\title{
DÜBLIN
}

Technological University Dublin

ARROW@TU Dublin

Articles

School of Food Science and Environmental

Health

2011-01-01

\section{Modelling the Effect of Asparaginase in Reducing Acrylamide Formation in Biscuits}

\author{
Monica Anese \\ University of Udine, monica.anese@uniud.it \\ Barbara Quarta \\ University of Udine, barbara.quarta@uniud.it \\ Jesus Maria Frias \\ Technological University Dublin, Jesus.Frias@tudublin.ie
}

Follow this and additional works at: https://arrow.tudublin.ie/schfsehart

Part of the Agriculture Commons, and the Food Chemistry Commons

\section{Recommended Citation}

Anese, M., Quarta, B. and Frias, J.M. (2011) Modelling the Effect of Asparaginase in Reducing Acrylamide Formation in Biscuits, Food Chemistry, Vol. 126, no. 2, 15, Pages 435-440 doi:10.1016/

j.foodchem.2010.11.007

This Article is brought to you for free and open access by the School of Food Science and Environmental Health at ARROW@TU Dublin. It has been accepted for inclusion in Articles by an authorized administrator of ARROW@TU Dublin. For more information, please contact arrow.admin@tudublin.ie, aisling.coyne@tudublin.ie, gerard.connolly@tudublin.ie.

Funder: COST Action 927:Thermally processed foods:

possible health implications

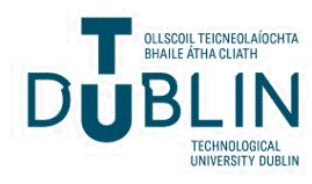


1 Modelling the effect of asparaginase in reducing acrylamide

\section{2 formation in biscuits}

3

4 Monica Anese $^{\mathrm{a}^{*}}$, Barbara Quarta ${ }^{\mathrm{a}}$, Jesus Frias ${ }^{\mathrm{b}+}$

5 a Dipartimento di Scienze degli Alimenti, University of Udine, Via Sondrio 2/A, 33100

6 Udine, Italy

$7{ }^{\mathrm{b}}$ School of Food Science and Environmental Health, Dublin Institute of Technology,

8 Cathal Brugha Street, Dublin 1, Ireland

9

10

11

12

13

14

15

16

17

18

19

20

21

$22{ }^{*}$ Corresponding author for the asparaginase treatments and chemical analysis

$23{ }^{+}$Corresponding authors for the data modelling

24

25 
27 The influence of asparaginase on acrylamide formation as well as colour development 28 in short dough biscuits was studied. In particular, asparaginase concentration, 29 incubation time and temperature were changed according to an experimental design. As

30 acrylamide formation resulted to vary significantly between biscuits obtained by using 31 the same ingredients and process, a mixed effect model was used to model variation of 32 acrylamide concentration. By contrast a fixed effect model was used for colour 33 polynomial analysis. Within the range of study, the overall results allowed to find the 34 best conditions to minimise acrylamide formation. It can be suggested that acrylamide 35 development is minimum at intermediate asparaginase concentrations and lowest time 36 and temperature of incubation. Asparaginase addition did not affect significantly the 37 colour of the final product, although the quadratic term of the incubation temperature 38 slightly did.

40 Keywords: Acrylamide, Asparaginase, Biscuits, Colour, Modelling. 


\section{Introduction}

52 Acrylamide is a toxic and probably human carcinogen molecule (IARC, 1994) that can form in heated foods as a consequence of the reaction between asparagine and a carbonyl source via Maillard-type reactions (Mottram, Wedzicha, \& Dodson, 2002; Stadler et al., 2002; Zyzak et al., 2003; Becalski, Lau, Lewis, \& Seaman, 2003; Yaylayan \& Stadler, 2005). Important acrylamide dietary sources are staple foods such as potato derivatives, cereal products and coffee (IRMM, 2005; FDA, 2006). Many potential routes have been identified to reduce acrylamide levels in foods. These are relevant to agronomical and technological strategies (CIAA, 2009). Among the latter

60 are interventions based on precursor consumption, i.e. fermentation and asparaginase 61 pre-treatments.

62 Asparaginase pre-treatment of raw potatoes and doughs has been claimed to reduce effectively acrylamide levels without altering the appearance and taste of the final product (Zyzak et al., 2003; Ciesarová, Kiss, \& Boegl, 2006; Hendriksen, Stringer, Ernst, Held-Hansen, Schafermayer, \& Corrigan, 2006; Ciesarová, Kukurová, Bednáriková, Marková, \& Baxa, 2009; Kukurová, Morales, Bednáriková, \& Ciesarová, 2009; Capuano et al, 2009; Ciesarová, Kukurová, \& Benešová 2010). Also, the effectiveness of asparaginase in reducing acrylamide is testified by a number of patent applications concerning different processed foods, such as snack foods, chips, dough foods, etc. (Elder, Fulcher, \& Leung, 2006; Elder, Fulcher, Kin-Hang Leung, \& Topor, 2007; Corrigan, 2008; de Boer, 2008). As it is well known asparaginase catalyzes the hydrolysis of asparagine into aspartic acid and ammonia, thereby specifically removing a key precursor for acrylamide formation. The commercial enzyme based on cloning of Aspergillus oryzae has received the generally recognized as safe status from the US and has been given a favourable evaluation by the Joint FAO/WHO Expert Committee on 
76 Food Additives (JECFA, 2007). It is now permitted for use in the United States,

77 Australia, New Zealand, and Denmark. In Canada, where enzymes used in food applications may be considered food additives, amendments to the Regulations that will allow the use of asparaginase as a food additive has been proposed (Health Canada, 2009). In 2008, the Standing Committee on the Food Chain and Animal Health authorised the use of asparaginase for bakery product manufacturing in two EU Member States, as a processing aid, since the manufactures have stated that the enzyme is inactivated during heat processing such as baking (SANCO, 2008).

Studies have shown that asparaginase activity is affected by enzyme dose, reaction time, temperature and $\mathrm{pH}$ at which the reaction occurs (Hendriksen, Kornbrust, Østergaard, \& Stringer, 2009). In particular, the A. oryzae asparaginase was shown to be most active in the neutral $\mathrm{pH}$ range and at temperatures up to $60{ }^{\circ} \mathrm{C}$. Furthermore, the enzyme activity is influenced by the contact with the substrate. In fact, a limited mobility of substrate and enzyme would be responsible for incomplete hydrolysis of asparagine and only partial reduction in acrylamide formation. In this regard the food decompartimentalisation as well as the water content of the reaction environment could greatly affect asparaginase efficacy in reducing acrylamide formation. In fact, great reductions of acrylamide content could be achieved by using relatively low asparaginase concentrations in formulated foods (up to $1000 \mathrm{U} / \mathrm{kg}$ ), such as bakery products and potato-based snacks, as a good enzyme distribution in the system can be reached. On the contrary, very high concentrations of asparaginase (>10000 U/L of pre-frying dipping solution) are necessary to obtain a significant reduction of acrylamide in fried potatoes (Pedreschi, Kaack, \& Granby, 2008). However, also in this case, any technological operation which favours the substrate diffusion and its contact with the enzyme can lead to a greater reduction of acrylamide levels. This is the case of potato pieces treated with 
101 asparaginase after blanching (Hendriksen et al., 2009). In fact, blanching reduces the 102 integrity of the potato, weakening the cell wall and membrane thereby improving the 103 substrate-enzyme contact. Besides, especially when asparaginase is added to food 104 formulations, the water content should be sufficiently high for mobility of reactants and

105 facilitate the contact between enzyme and substrate (Amrein, Schoenbaechler, Escher, 106 \& Amadò, 2004; Hendriksen et al., 2009). For this reason the enzyme resulted more 107 effective when added in the aqueous phase of the dough preparation instead of in the 108 mixture.

109 No fundamental modelling of the effect of asparaginase on this process is available.

110 Due to the complexity of the relationship between the environmental variables involved

111 in the enzymatic process and acrylamide formation, ii) the effective enzymatic activity 112 and iii) the final acrylamide concentration achieved, a study of these relationship is 113 needed to find the best conditions to minimise acrylamide formation.

114 Natural variability in baking processes is an influencing factor and acrylamide 115 formation has been seen to vary significantly between items with similar ingredients and 116 cooking procedures (Levine \& Smith, 2005). Evenmore, Bråthen and Knutsen (2005)

117 showed how variability played an important role in starch systems affecting the final 118 value of acrylamide after a baking process. If this process is to be scaled-up this 119 variability needs to be assessed so that tolerances may be established within the normal 120 ranges of variability of an industrial process (Aguirre, Frias, Barry-Ryan, \& Grogan, 121 2008).

122 The aim of this study was to investigate the influence of asparaginase in reducing 123 acrylamide formation in short dough biscuits. The effect of asparaginase addition on 124 browning development was also investigated. In particular, asparaginase concentration, 
125 incubation time and temperature were modulated according to a three variable, three

126 level central composite design.

127

\section{2. Materials and methods}

129 2.1. Sample preparation

130 Short dough biscuits were prepared according to the slightly modified formulation by

131 Gallagher, Kenny and Arendt (2005). The formulation consisted of flour, margarine

132 (Unigrà, Italy), sucrose (Carlo Erba, Milano Italy), water, glucose (Carlo Erba, Milano

133 Italy), salt (Carlo Erba, Milano Italy), asparagine (Sigma-Aldrich, Italy) and baking

134 powder (sodium hydrogen carbonate, disodium diphosphate, dried starch) (Cameo,

135 Italy). The non-flour ingredients were added to the recipe at 40,35, 20, 5, 0.7, 0.1 and

$1360.5 \%$ flour weight, respectively. Different levels of asparaginase (Novozymes A/S,

137 Denmark, $3500 \mathrm{U} / \mathrm{g}$ ) in the range from $100 \mathrm{U} / \mathrm{kg}$ of flour to $900 \mathrm{U} / \mathrm{kg}$ of flour were

138 added to this recipe according to a three-factor, three-level face centered cube central

139 composite design (CCD) (Table 1). To assure a homogeneous distribution in the dough,

140 asparaginase was dispersed in the aqueous phase before to be added to the dry

141 ingredients. After mixing and a 30 min resting time at $4{ }^{\circ} \mathrm{C}$, the dough was sheeted to

$1420.3 \mathrm{~cm}$ thickness, cut to a diameter of $7 \mathrm{~cm}$ and left in a thermostatic cell at different

143 incubation temperatures and times according to the CCD (Table 1). In particular, the

144 incubation temperature ranged from $20{ }^{\circ} \mathrm{C}$ to $54{ }^{\circ} \mathrm{C}$ and the incubation time from 10

$145 \mathrm{~min}$ to $30 \mathrm{~min}$. According to literature data, the enzyme is active within these variable

146 ranges (Hendriksen et al., 2009). In addition, the chosen temperature and reaction time

147 may roughly correspond to the resting temperatures and time generally applied for the

148 dough at industrial level. The samples were baked in an air-circulating oven (Salvis

149 Thermocenter, Oakton, Vernon Hills, IL, USA) at $200{ }^{\circ} \mathrm{C}$ up to a final moisture of $2 \%$. 
150 Biscuits prepared without asparaginase addition were taken as a control. In order to assess the variability of this process, simulating an industrial situation, this process was repeated with 6 different batches of dough biscuits.

\subsection{Analysis of acrylamide}

155 Acrylamide determination was carried out according to the method of Anese,

156 Bortolomeazzi, Manzocco, Manzano, Giusto and Nicoli (2009). Briefly, $1000 \mu \mathrm{L}$ of an 157 aqueous solution of $2,3,3\left[{ }^{2} \mathrm{H}_{3}\right]$ acrylamide ( $\mathrm{d}_{3}$-acrylamide) $(0.20 \mu \mathrm{g} / \mathrm{mL})$ (Isotec, Sigma158 Aldrich, Italy) as internal standard and $15 \mathrm{~mL}$ of water Milli Q (Millipore, Italy) were 159 added to $1 \mathrm{~g}$ of finely ground biscuit weighed into a $100 \mathrm{~mL}$ centrifuge tube. After 160 extraction at $60{ }^{\circ} \mathrm{C}$ for 30 min under magnetic stirring, the mixture was centrifuged at $16112000 \mathrm{x}$ g for $15 \mathrm{~min}$ at $4{ }^{\circ} \mathrm{C}$ (Beckman, Avanti Centrifyge J-25, Palo Alto, CA, USA). 162 Aliquots of $10 \mathrm{~mL}$ of the clarified aqueous extract were cleaned-up by solid phase extraction (SPE) on an Isolute Env+, $1 \mathrm{~g}$ (Biotage, Sweden). The volume of the eluted

164 fraction was reduced under vacuum, to about $1.5-2 \mathrm{~mL}$ by using a rotary evaporator at 165 a temperature of $80{ }^{\circ} \mathrm{C}$ and filtered through a $0.45 \mu \mathrm{m}$ membrane filter before the HPLC-MS analysis. LC-ESI-MS-MS in positive ion mode analyses were performed by

167 a Finnigan LXQ linear trap mass spectrometer (Thermo Electron Corporation, San Josè,

168 CA, USA) coupled to a Finnigan Surveyor LC Pump Plus equipped with a thermostated 169 autosampler and a thermostated column oven. The analytical column was a Waters 170 Spherisorb ODS2 $(250 \times 2.0 \mathrm{~mm}, 5 \mu \mathrm{m})$. Elution was carried out at a flow-rate of 0.1

$171 \mathrm{~mL} / \mathrm{min}$, in isocratic conditions, at $30{ }^{\circ} \mathrm{C}$ using as mobile phase a mixture of $98.9 \%$ 172 water, $1 \%$ methanol and $0.1 \%$ formic acid (v/v/v). Full scan MS/MS was carried out by 173 selecting the ions at $m / z, 72$ and $m / z, 75$ as precursor ions for acrylamide and $\mathrm{d}_{3^{-}}$ 174 acrylamide respectively. The area of the chromatographic peaks of the extracted ion at 
$175 \mathrm{~m} / z$ 55, due to the transition $72>55$, and at $m / z$ 58, due to the transition $75>58$ were

176 used for the quantitative analysis. The quantitative analysis was carried out with the

177 method of the internal standard. The relative response factor of acrylamide with respect

178 to $\mathrm{d}_{3}$-acrylamide was calculated daily by analyzing a standard solution. For each run,

179 analyses were made in duplicate on six replicated experiments. Acrylamide

180 concentration was expressed as ng/g of dry matter.

181

182 2.3. Analysis of colour

183 Colour analysis was carried out on sample surface using a tristimulus colorimeter

184 (Chromameter-2 Reflectance, Minolta, Osaka, Japan) equipped with a CR-300

185 measuring head. The instrument was standardized against a white tile before

186 measurements. Colour was expressed in L* (lightness/darkness), a* (redness/greenness)

187 and $b^{*}$ (yellowness/blueness) scale parameters. The total colour change in the $\mathrm{L}^{*}, \mathrm{a}^{*}, \mathrm{~b}^{*}$

188 colorimetric space, $\Delta E^{*}$, was then calculated from Eq. (1) (Clydesdale, 1978):

189

190

$\Delta E^{*}=\left(\left(\left(L^{*}-L_{0} *\right)^{2}+\left(a^{*}-a_{0} *\right)^{2}+\left(b^{*} \cdot b_{0} *\right)^{2}\right)^{1 / 2}\right)$

Eq. (1)

191

192

where $L^{*}, a^{*}, b^{*}$ are the actual colour values and $L_{0} *, a_{0} *$ and $b_{0} *$ are the colour values

193 for a control sample, i.e. obtained without asparaginase addition.

194 For each run, analyses were made at least in triplicate on three replicated experiments.

195

196 2.4. Determination of total solid content

197 Total solid content was determined by gravimetric method by drying the samples in a

198 vacuum oven $(1.32 \mathrm{kPa})$ at $75^{\circ} \mathrm{C}$ until a constant weight.

199 
201 Modelling was aimed at describing the variation of acrylamide concentration and colour data as a function of the variables of the central composite design.

203 In the case of acrylamide analysis, coded variables were used to model this variation:

204

205

$x=\frac{x-\bar{x}}{\Delta x / 2}$

Eq. (2)

206

207

where $\mathrm{x}$ is the explanatory variable normalised, $\bar{x}$ is the average of the variable and

$\Delta x$ is the range between the máximum and mínimum value of $\mathrm{x}$.

The lme and lme4 libraries of the R software package (R Development Core Team, 2009) were used to fit a mixed effects model with the following components:

1. A fixed effect component containing a second order polynomial model to the dependant variables.

2. A random effect component that contains the effect of variability on the

215

216 This resulted in the following model:

217

218

$y=B_{0}+\sum B_{i} x_{i}+\sum B_{i i} x_{i}^{2}+\sum B_{i j} x_{i} x_{j}+Z b+\varepsilon, \varepsilon \sim \mathrm{N}\left(0, \sigma^{2}\right), b \sim \mathrm{N}\left(0, \sigma^{2} \mathrm{E}\right)$ Eq. (3)

220 where $B_{0}$ is a constant and $B_{i}, B_{i i}, B_{i j}$ are regression coefficients of the model and $x_{i}$ and

$221 x_{j}$ are independent variables in coded values. The vector $\mathrm{b}$ represents the random effects,

222 i.e. variations due to random nature associated to a $\mathrm{Z}$ model matrix with a relative

223 variance-covariance matrix $\mathrm{E}$ which contains possible predictors influencing the 
224 variability of the acrylamide concentration. $\mathrm{N}$ denotes the multivariate normal 225 distribution (Pinheiro \& Bates, 2000).

226 In the case of colour analysis, Statistica for Windows (Statsoft Inc., 1993) was used to

227 fit the second order models to the dependant variables using the following equation:

$$
y=B_{0}+\sum B_{i} x_{i}+\sum B_{i j} x_{i} x_{j}+\sum B_{i i} x_{i}^{2} \quad \text { Eq. (4) }
$$

230 The criteria for eliminating a variable from the full regression equation was based on

$231 \mathrm{R}^{2}$, standard error to estimate ( $\mathrm{SE}$ ) and significance F-test (and the derived $\mathrm{p}$ values).

\section{Results and discussion}

234 Table 2 shows acrylamide mean values and the corresponding standard deviations of 235 short dough biscuits added with asparaginase according to the CCD. For each run of the $236 \mathrm{CCD}$, the results of acrylamide concentration were based on two replicated analyses on 237 six different batches of short dough biscuits, i.e. made using the same recipe and process. Although repetitions within each batch were good with standard deviations ranging from 0.1 to 10 , results among batches of each run varied greatly. For instance,

240 the acrylamide levels in asparaginase-treated made with $500 \mathrm{U} / \mathrm{kg}$ of flour and a $20 \mathrm{~min}$

241 incubation time at $20^{\circ} \mathrm{C}$ (run 12) ranged from 49 to $120 \mathrm{ng} / \mathrm{g}_{\mathrm{dm}}$, with an average of 90

$242 \mathrm{ng} / \mathrm{g}_{\mathrm{dm}}$ and a coefficient of variation of $25 \%$. Similar experimental variations were 243 found by Hendriksen et al. (2009) in semisweet biscuits. The variation components

244 arising from the analysis of acrylamide or from replicates of the same experimental 245 conditions with the same batch of biscuit were not big enough to contribute to this $25 \%$ 246 of variation and therefore it was concluded that the origin of this arised from the 
247 preparation and processing of different batches of biscuit. In the light of a possible

248 variation between batches of biscuits been prepared, data were analysed by using a 249 mixed effect model (Pinheiro \& Bates, 2000). The two components of the model were

250 a) fixed effects, that included all the effects of the variation of the dependent variables

251 (asparaginase dose, incubation temperature and time) which affect the acrylamide 252 concentration in a statistically significant manner; b) random effects, which include the 253 variation in final acrylamide concentrations that are due to changes among batches of 254 the biscuit dough.

255 Table 3 shows the result of the polynomial mixed effect regression. All non-significant

256 fixed terms were deleted form the model following an iterative process until the model 257 was satisfactory. As it can be seen in the table there are two quadratic effects, pointing 258 to two variables with possible minima-maxima. No significant correlation between 259 estimates was found, indicating that these parameter estimates and the standard errors 260 were estimated accurately. Fig. 1 diagnostic plots indicate that the residuals variance 261 was constant and that it followed approximately a normal distribution.

262 Fig. 2 shows the importance of the different regression variables in the acrylamide 263 formation. As it can be seen, within the margins of the present study, the variable that 264 has a bigger effect is the concentration of asparaginase introduced, followed by the 265 temperature effect. The incubation time of the asparaginase infused dough seems to be 266 the least important variable, being found mainly at the bottom of the Pareto chart. This

267 points to the result that within the present conditions, variations in temperature and/or 268 asparaginase will affect a bigger change in the final acrylamide concentration of the 269 biscuits. These results are in agreement with those reported by Hendriksen et al. (2009) 270 for semisweet biscuits incorporated with asparaginase. Models including random effects 
271 depending on the temperature, asparaginase inoculation and incubation time were built,

272 however none of them contributed to increase the quality of the fit, as the log-

273 likelihood ratio test did not proof significant for any of the models $(p<0.05)$. As a result,

274 the batch-to-batch variation was modelled as a random intercept process. The

275 importance of this random process can be seen in the magnitude of the standard

276 deviation of it compared to any of the normalised coefficients in the model.

277 The batch-to-batch variability can be attributed to a non homogeneous distribution of 278 the enzyme in the dough. This in turn can be ascribable either to the fact 279 that very small quantities of the enzyme are incorporated to 280 the other ingredients of the dough so that it cannot be uniformly 281 distributed, or to a matrix effect. In the latter case, it can be suggested that the presence 282 of fat contributes to create hydrophobic zones where the activity of the aqueous 283 enzymatic suspension is limited.

284 The matrix effect could also explain the lower average percentage reductions of 285 acrylamide levels due to asparaginase activity as compared to most of the data reported 286 in the literature. In fact, we found that asparaginase contributed to reduce acrylamide 287 concentration by 27 to $70 \%$ (Table 4), while the reductions reported in the literature are 288 on average higher than 85-90\% (Zyzak et al., 2003; Ciesarová et al., 2006; Kukurová et 289 al., 2009; Capuano et al., 2009). These differences in asparaginase efficiency can be 290 attributable to the different complexity from the compositional standpoint of the 291 systems considered. In fact, the above mentioned papers deal with food model systems, 292 i.e. made with starch, sugar and water, whereas, in the present study, biscuits obtained 293 by using additional ingredients, such as fat and salt, were considered. It must be pointed 
294 out that our results are in agreement with those of Hendriksen et al. (2009), who also

295 considered real food systems.

296 From Table 3 are two possible minima of acrylamide, in the asparaginase concentration

297 and with the incubation time. In Fig. 3 and 4 contour plots of the acrylamide in respect

298 of the experiment variables are presented. It can be seen that there is a minimum of 299 acrylamide formed at intermediate asparaginase concentrations (500 U/kg of flour) the 300 lowest incubation time and temperature.

301 It is interesting to note how high values of asparaginase seem to increase greatly the

302 final amount of acrylamide found and that excessive asparaginase inoculation followed

303 by high incubation times may actually increase the average acrylamide in the biscuits,

304 in comparison with low additions of asparaginase. However, within the range of the

305 study, if the final inoculums of asparaginase is well controlled and the incubation

306 temperature is kept to a minimum, it is reasonable to admit that there will be a decrease

307 of acrylamide concentration in the biscuit no matter what the incubation time will be.

308 Fig. 5 shows a stochastic simulation of the final levels of acrylamide under $500 \mathrm{U} / \mathrm{kg}$ of 309 asparaginase with $10 \mathrm{~min}$ of incubation time at $20{ }^{\circ} \mathrm{C}$, which are the conditions

310 providing a minimum acrylamide concentration in the region of study. It can be seen

311 that even with the variation between batches of biscuits, the optimal conditions can

312 ensure that concentrations of acrylamide will not go over $138 \mathrm{ng} / \mathrm{g}_{\mathrm{dm}}$ with a coverage of

$31395 \%$ of all batches. This is in contrast with the worse conditions observed (maximum

314 asparaginase added, maximum temperature of incubation at the shortest incubation

315 time) which produce an acrylamide $282 \mathrm{ng} / \mathrm{g}_{\mathrm{dm}}$ with coverage of $95 \%$ of all the batches 316 of bread and samples. 
317 Besides its feasibility and compatibility with the existing industrial process, acrylamide

318 mitigation in biscuits by means of asparaginase addition has to take into account its

319 effects on the sensory properties of foods. To this regard, the effect of asparaginase

320 addition on the colour of the biscuits was studied. The results of the polynomial

321 regression of total colour change data are shown in Table 5. In this case the mixed effect

322 modelling was not included in the design because the colour data variability could be

323 assigned to a single experimental error term. A random distribution of residuals was

324 found. As it can be seen, the total colour change was significantly affected only by the

325 quadratic term of the incubation temperature, while neither the asparaginase

326 concentration nor the time influenced this parameter. This result is in agreement with

327 observations made by other Authors who did not found any significant change in colour

328 between asparaginase untreated and treated food systems (Capuano et al., 2009;

329 Kukurová et al., 2009). It is likely that the temperature adopted during dough incubation

330 may influence the development of non-enzymatic browning reactions: the higher the

331 incubation temperature the higher the rate of the reaction that leads to the formation of

332 brown molecules. It is worth to noting that, due to the low temperature estimate value,

333 in the range of incubation temperatures compatible with the enzyme activity (20 to 60

$334{ }^{\circ} \mathrm{C}$ ), the total colour change can vary in a narrow range of values, i.e. between 2 and 8 .

\section{Conclusions}

337 This study showed a strategy to assess the benefit of asparaginase in reducing the

338 acrylamide formation in biscuits for managing the industrial process. The model used in

339 this study allowed for the assessment of the effect of the natural variability associated to

340 the baking process in final acrylamide concentration, besides the variation of the

341 selected dependent variables. It can be concluded that, within the range of study, an 
342 intermediate asparaginase concentration of $500 \mathrm{U} / \mathrm{kg}$ combined with the lowest time and

343 temperature of incubation effectively reduced acrylamide formation in short dough

344 biscuits without affecting the colour of the final product.

\section{Acknowledgements}

347 This work was supported by the COST Action 927 "Thermally processed foods: 348 possible health implications".

\section{References}

351

Aguirre, L., Frías, J.M., Barry-Ryan, C., \& Grogan, H. (2008) Assessing the effect of product variability on the management of the quality of mushrooms (Agaricus bisporus), Postharvest Biology and Technology, 49, 247-254.

Amrein, T. M., Schoenbaechler, B., Escher, F., \& Amadò, R. (2004). Acrylamide in gingerbread: critical factors for formation and possible ways for reduction. Journal of Agricultural and Food Chemistry, 52, 4282-4288.

Anese, M., Bortolomeazzi, R., Manzocco, L., Manzano, M., Giusto, C., \& Nicoli, M. C. (2009). Effect of chemical and biological dipping on acrylamide formation and sensory properties in deep-fried potatoes. Food Research International, 42, 142147.

Becalski, A., Lau, B. P-L., Lewis, D., \& Seaman, S. W. (2003). Acrylamide in foods: occurrence, sources and modelling. Journal of Agricultural and Food Chemistry, $51,802-808$. 
364 Bråthen, E., \& Knutsen S. H. (2005). Effect of temperature and time on the formation of acrylamide in starch-based and cereal model systems, flat breads and bread. Food Chemistry, 92, 693-700.

367 Capuano, E., Ferrigno, A., Acampa, I., Serpen, A., Açar, O. Ç., Gökmen, V., \& Fogliano, V. (2009). Effect of flour type on Maillard reaction and acrylamide formation during toasting of bread crisp model systems and mitigation strategies. Food Research International, 42, 1295-1302.

CIAA (2009). The CIAA Acrylamide Toolbox. Confederation of the Food and Drink Industries. <www.ciaa.be>.

Ciesarová, Z., Kiss, E., \& Boegl, P. (2006). Impact of L-asparaginase on acrylamide content in potato products. Journal of Food Nutrition and Research, 45, 141-146.

375 Ciesarová, Z., Kukurová, K., Bednáriková, A., Marková, L., \& Baxa, S. (2009). Improvement of cereal product safety by enzymatic way of acrylamide mitigation. Czech Journal of Food Science, 27, 96-98.

Ciesarová, Z., Kukurová, K., \& Benešová, C. (2010). Enzymatic elimination of acrylamide in potato-based thermally treated foods. Nutrition \& Food Science, 40,

Clydesdale, F. M. (1978). Colorimetry-Methodology and applications. Critical Reviews in Food Science and Nutrition, 10, 243-301.

Corrigan, P. J. (2008). Methods for reducing asparagine in a dough food component using water activity. Patent No US20080166450-A1.

385 de Boer, L. (2008). Novel process for enzymatic acrylamide reduction in food products. Patent No EP1896576-A1.

387 Elder, V. A., Fulcher, J. G., \& Leung, H. K. H. (2006). Method for reducing acrylamide formation in thermally processed foods. Patent No US20060127534-A1. 
Elder, V. A., Fulcher, J. G., Kin-Hang Leung, H., \& Topor, M. G. (2007). Methods for reducing acrylamide formation. Patent No US20070141225-A1.

391 FDA, Center for Food Safety and Applied Nutrition (2006). <http://www.cfsan.fda.gov/ lrd/pestadd.html\#acrylamide>.

393 Gallagher, E., Kenny, S., \& Arendt, E. K. (2005). Impact of dairy protein powders on biscuit quality. European Food Research and Technology, 221, 273-243.

395 Health Canada (2009). <http://www.hc-sc.gc.ca/fn-an/consultation/init/_2009asparaginase/prop-asparaginase-eng.php>.

Hendriksen, H. V., Stringer, M., Ernst, S., Held-Hansen, P., Schafermayer, R., \& Corrigan, P. (2006). Novoenzymes A/S. Patent No WOO6053563.

Hendriksen, H. V., Kornbrust, B. A., Østergaard, P. R., \& Stringer, M. A. (2009). 400 Evaluating the potential for enzymatic acrylamide mitigation in a range of food 401 products using an asparaginase from Aspergillus oryzae. Journal of Agricultural

IARC (1994). IARC Monographs on the Evaluation of Carcinogenic Risks to Humans (Vol. 60). Lyon, France: International Agency for Research on Cancer.

IRMM (2005). EU Database on Acrylamide Levels in Food. <www.irmm.jrc.be/htlm/acrivities/acrylamide/database.htm>.

JEFCA (2007). Compendium of Food Additive Specifications. No. 4 FAO JECFA Monographs, Joint FAO/WHO Expert Committee on Food Additives. 409 <www.fao.org/ag/jefca-additives/specs/monograph4/additive-498-m4.pdf>

410 Kukurová, K., Morales, F., Bednáriková, \& Ciesarová, Z. (2009). Effect of L411 asparaginase on acrylamide mitigation in a fried-dough pastry model. Molecular Nutrition and Food Research, 53, 1532-1539. 
413 Levine, R. S., \& Smith, R. E. (2005). Sources of variability of acrylamide levels in 414 cracker model. Journal of Agricultural and Food Chemistry, 53, 4410-4416.

415 Mottram, D. S., Wedzicha, B. L., \& Dodson, A. (2002). Acrylamide is formed in the $416 \quad$ Maillard reaction. Nature, 419, 448-449.

417 Pedreschi, F., Kaack, K., \& Granby, K. (2008). The effect of asparaginase on 418 acrylamide formation in French fries. Food Chemistry, 109, 386-392.

419 Pinheiro, J., \& Bates D. M. (2000). Mixed-Effects Models in S and S-PLUS. New York, $420 \quad$ USA: Springer Verlag.

R Development Core Team (2009). R: A language and environment for statistical computing. R Foundation for Statistical Computing, Vienna, Austria. <www.Rproject.org>.

SANCO, Standing Committee on the Food Chain and Animal Health (2008). Summary Report by the Section of Toxicological Safety of the Food Chain, Brussels <http://ec.europa.eu/food/committees/regulatory/scfcah/toxic/summary11042008_e n.pdf>.

Stadler, R. H., Blank, I., Varga, N., Robert, F., Hau, J., Guy, P. A., Robert, M.-C., \& Riediker, S. (2002). Acrylamide from Maillard reaction products. Nature, 419, $449-450$.

431 Yaylayan, V. A., \& Stadler, R. H. (2005). Acrylamide formation in food: a mechanistic perspective. Journal of AOAC International, 88, 262-267.

Zyzak, D. V., Sanders, R. A., Stojanovic, M., Tallmadge, D. H., Loye Eberhart, B., 434 Ewald, D. K., Gruber, D. C., Morsch, T. R., Strothers, M. A., Rizzi, G. P., \& 435 Villagran, M. D. (2003). Acrylamide formation mechanism in heated foods. Journal of Agricultural and Food Chemistry, 51, 4782-4787. 


\section{Caption for figures}

443 Fig. 1. Residual against fitted values (left) and residual quantiles against standard 444 normal quantiles (right) plots of the polynomial mixed effects model.

445 Fig. 2. Effects plot for the fixed part of the polynomial model.

446 Fig. 3. Dependence of acrylamide concentration with asparaginase concentration and 447 incubation time at the lowest incubation temperature.

448 Fig. 4. Contour of the dependence of acrylamide concentration with the normalised 449 asparaginase concentration and incubation temperature at the lowest incubation time.

450 Fig. 5. Uncertainty assessment of the variability of acrylamide for the optimal conditions of intermediate asparaginase content, minimum incubation temperature and minimum incubation time. 


\section{Table 2}

Acrylamide mean values and corresponding standard deviations of short dough biscuits added asparaginase according to the $\mathrm{CCD}$

\begin{tabular}{|c|c|c|}
\hline Run & $\begin{array}{l}\text { Acrylamide concentration } \\
\qquad\left(\mathrm{ng} / \mathrm{g}_{\mathrm{dm}}\right)\end{array}$ & Standard deviation \\
\hline 1 & 142.1 & 59.8 \\
\hline 2 & 111.2 & 39.2 \\
\hline 3 & 200.4 & 40.3 \\
\hline 4 & 201.8 & 31.0 \\
\hline 5 & 137.0 & 43.4 \\
\hline 6 & 203.3 & 8.2 \\
\hline 7 & 229.1 & 25.3 \\
\hline 8 & 140.0 & 31.8 \\
\hline 9 & 143.1 & 32.6 \\
\hline 10 & 161.5 & 61.1 \\
\hline 11 & 223.9 & 4.6 \\
\hline 12 & 90.0 & 21.4 \\
\hline 13 & 135.9 & 3.9 \\
\hline 14 & 110.0 & 46.2 \\
\hline 15 & 101.1 & 12.3 \\
\hline
\end{tabular}




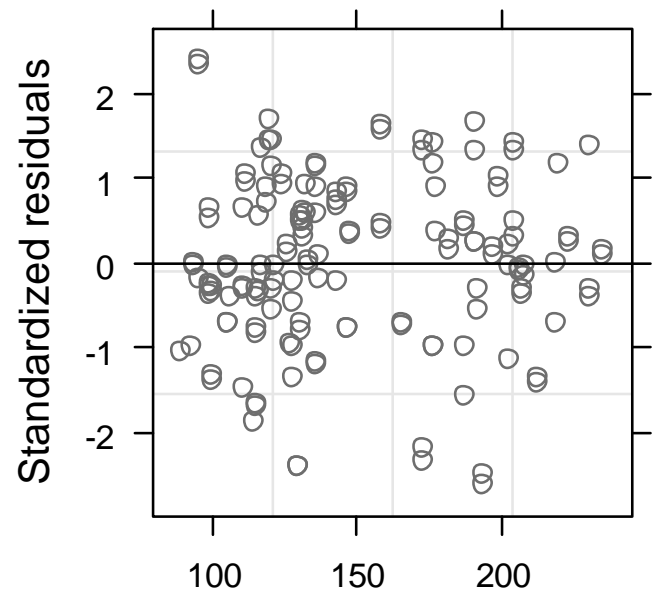

Fitted values

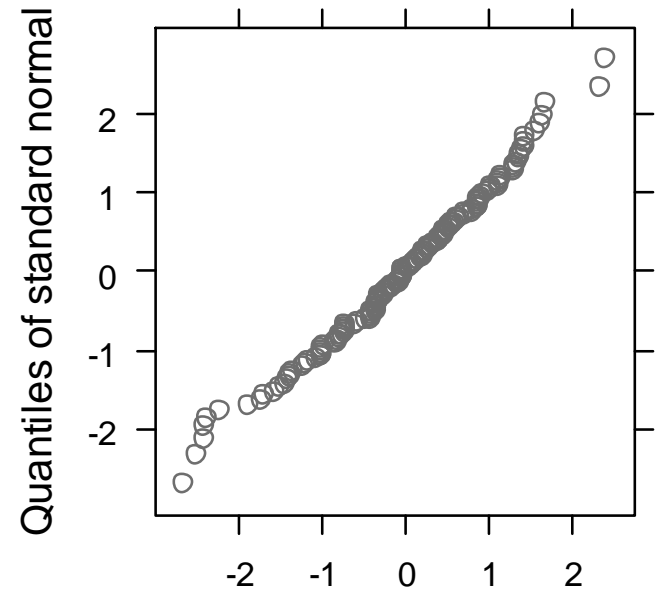

Standardized residuals

Fig. 6. Residual against fitted values (left) and residual quantiles against standard normal quantiles (right mixed effects model. 


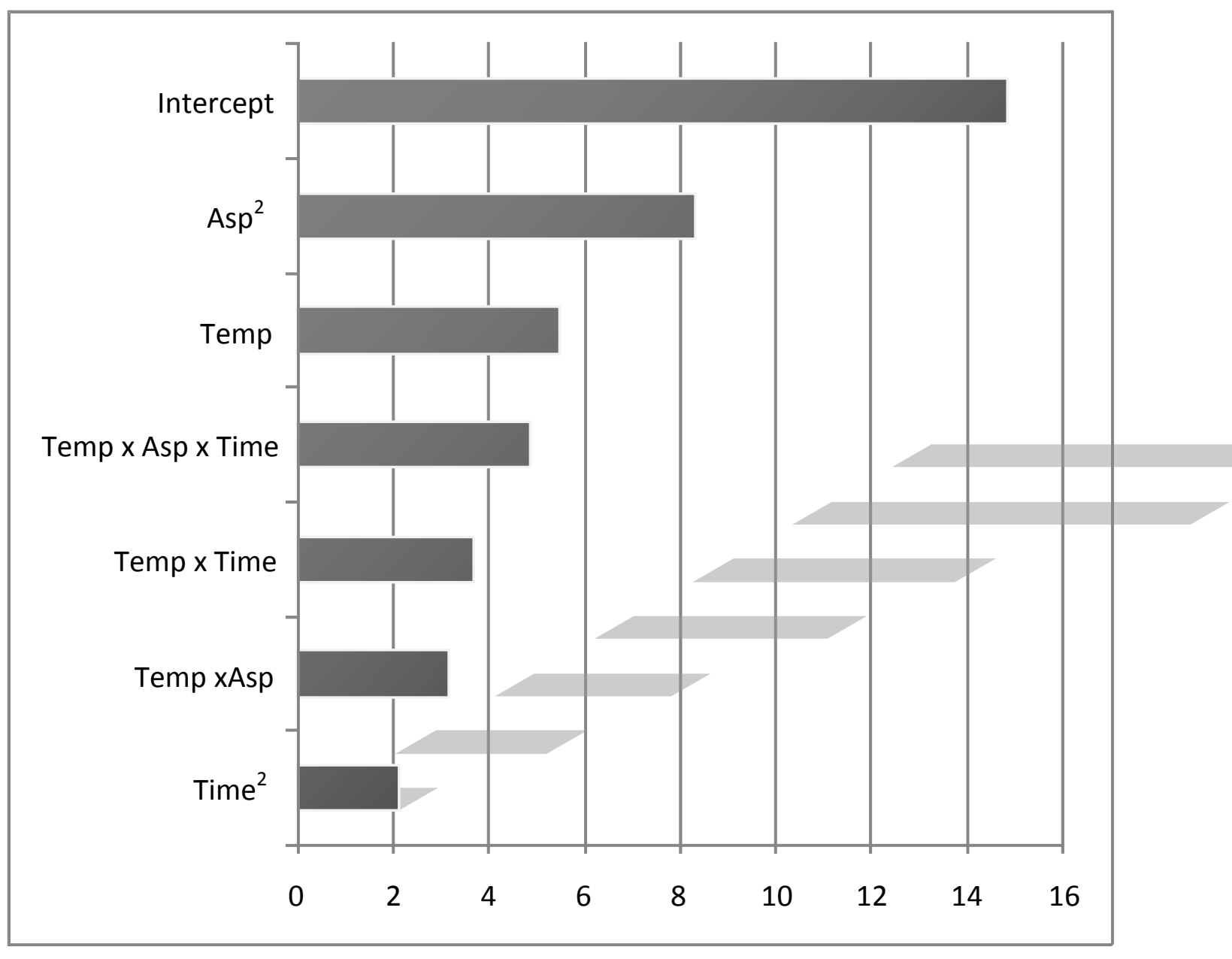

Fig. 7. Effects plot for the fixed part of the polynomial model. 


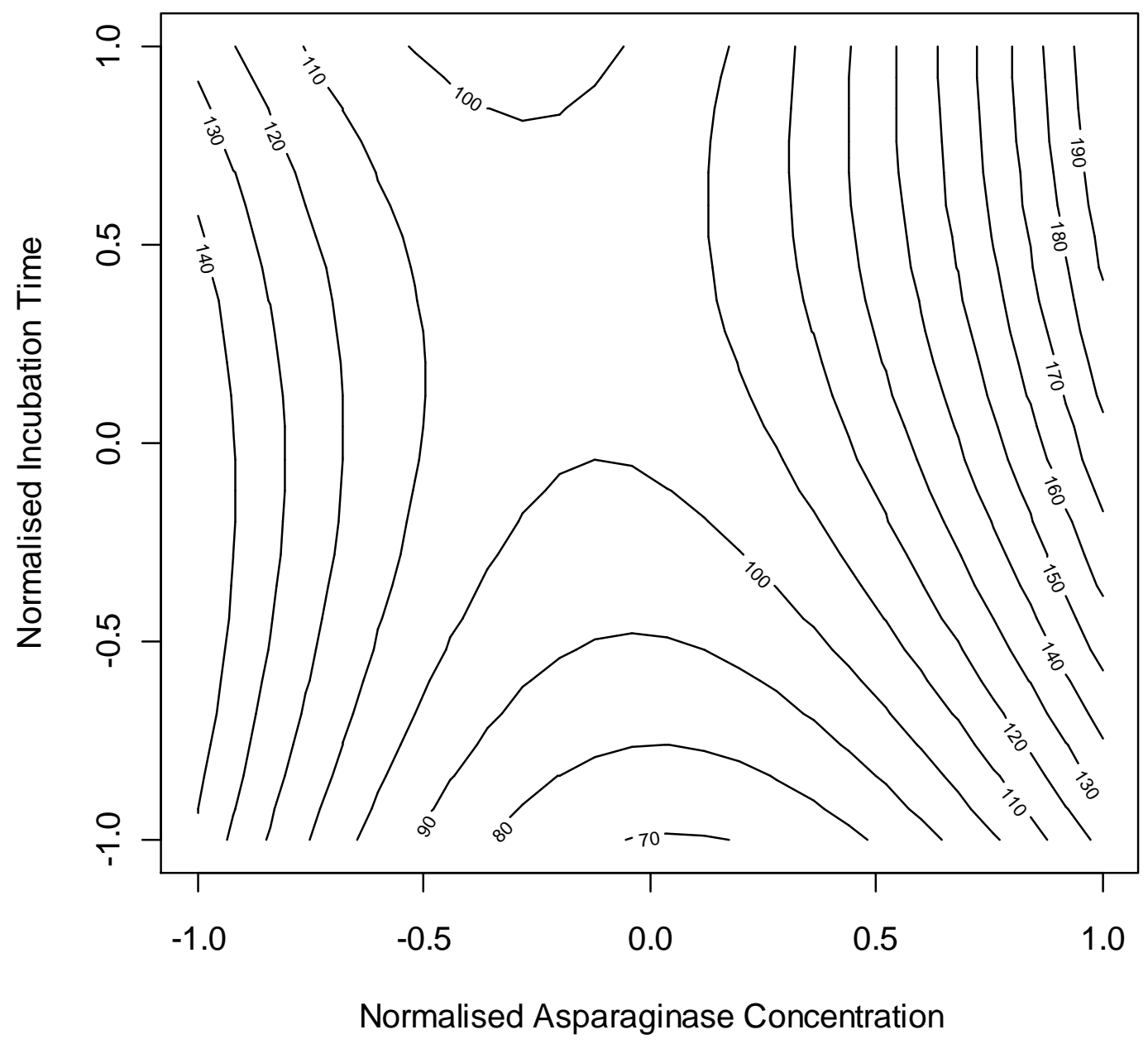

Fig. 8. Dependence of acrylamide concentration with asparaginase concentration and incubation time temperature. 


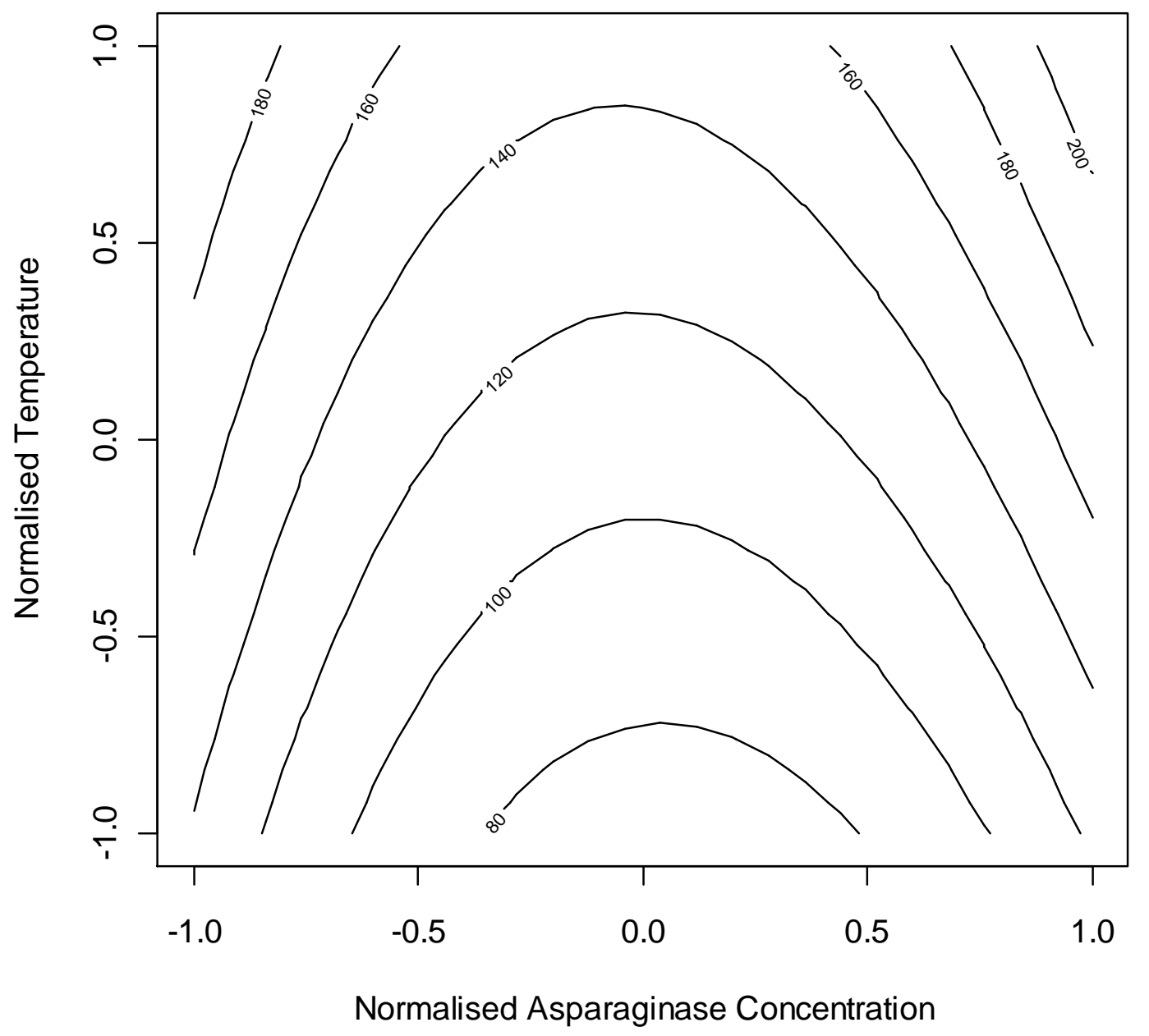

Fig. 9. Contour of the dependence of acrylamide concentration $\left(\mathrm{ng} / \mathrm{g}_{\mathrm{dm}}\right)$ with the normalised aspara incubation temperature at the lowest incubation time. 


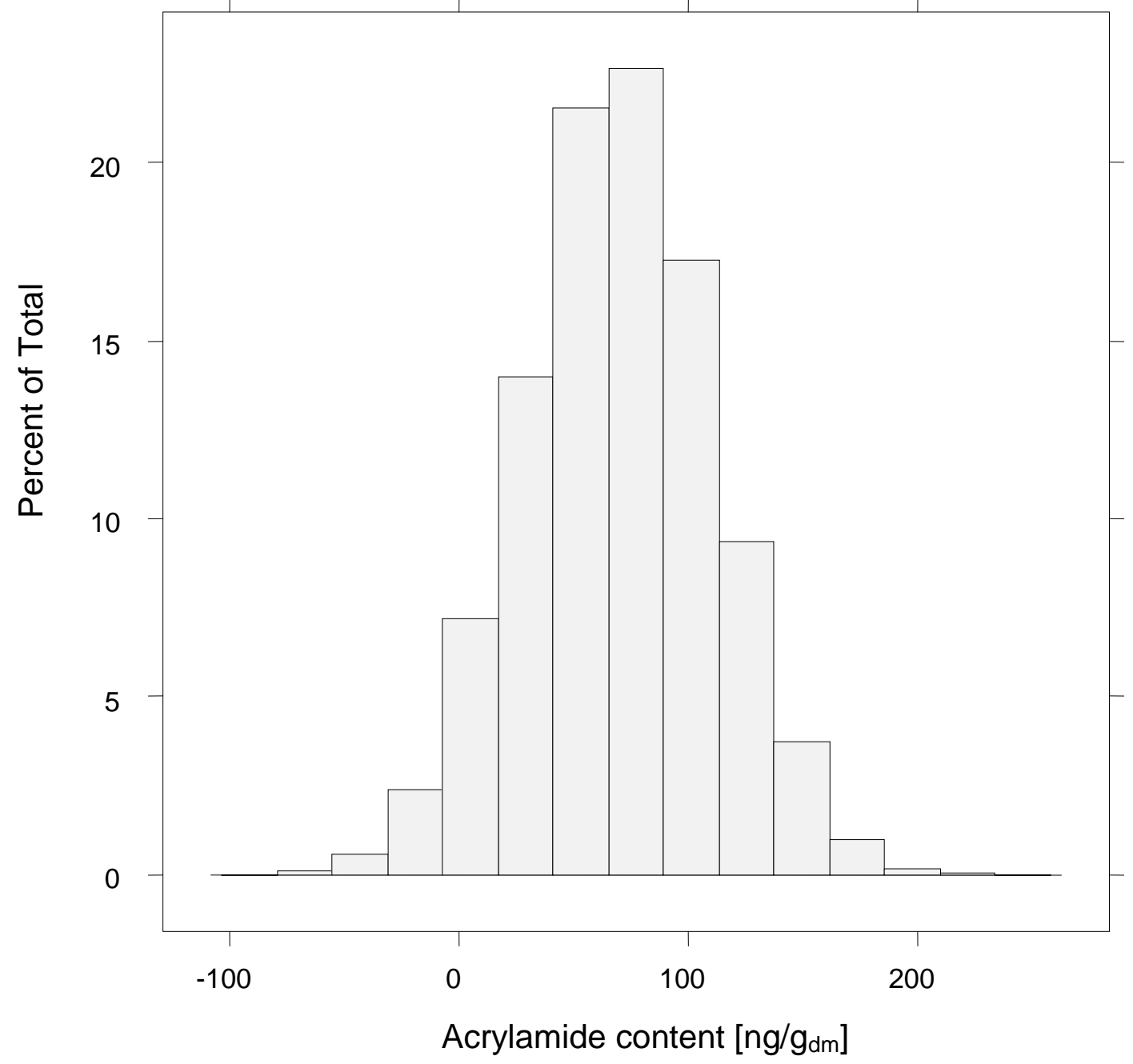

Fig. 10. Uncertainty assessment of the variability of acrylamide for the optimal conditions of intermedi minimum incubation temperature and minimum incubation time. 